\title{
Subcutaneous Abatacept for the Treatment of Rheumatoid Arthritis: Longterm Data from the ACQUIRE Trial
}

\author{
Mark C. Genovese, César Pacheco Tena, Arturo Covarrubias, Gustavo Leon, Eduardo Mysler, \\ Mauro Keiserman, Robert Valente, Peter Nash, J. Abraham Simon-Campos, Jane Box, \\ Clarence William Legerton III, Evgeny Nasonov, Patrick Durez, Ingrid Delaet, Julie Teng, \\ and Rieke Alten
}

ABSTRACT. Objective. Assess longterm tolerability, safety, and efficacy of subcutaneous (SC) abatacept (ABA) in methotrexate-refractory patients with rheumatoid arthritis (RA).

Methods. The phase III, multinational Abatacept Comparison of Sub[QU]cutaneous Versus Intravenous in Inadequate Responders to MethotrexatE (ACQUIRE) trial comprised a 6-month, randomized, double-blind (DB) period, in which patients received intravenous (IV) or SC ABA, plus MTX, followed by an open-label, longterm extension (LTE), in which patients received SC ABA, $125 \mathrm{mg} /$ week. Safety and efficacy from the LTE ( 3.5 yrs of exposure) are reported.

Results. Patients who completed the DB period (1372/1385, 99.1\%) entered the LTE; 1134 patients $(82.7 \%)$ kept taking the treatment at time of reporting. Mean (SD) was 31.9 months (6.8); median (range) exposure was 33.0 (8-44) months. Patients entering the LTE had longstanding, moderate-to-severe disease [mean 7.6 (7.9) yrs and DAS28 (C-reactive protein) $6.2(0.9)$ ]. Incidence rates (events/100 patient-yrs) were reported for serious adverse events $(8.76,95 \%$ CI 7.71, 9.95), infections $(44.80,95 \%$ CI 41.76, 48.01), serious infections $(1.72,95 \%$ CI 1.30, 2.27), malignancies $(1.19,95 \%$ CI $0.86,1.66)$, and autoimmune events $(1.31,95 \%$ CI $0.95,1.79)$. Twenty-seven patients (2\%) experienced injection-site reactions; all except 1 were mild. American College of Rheumatology 20,50, and 70 responses achieved during the DB period were maintained through the LTE, and on Day 981 were $80.2 \%$ (95\% CI 77.2, 83.2), 63.5\% (95\% CI 58.2, 68.9), and 39.5\% (95\% CI 34.0, 44.9) for patients who kept taking SC ABA, and 80.0\% (95\% CI 77.0, 83.0), 63.2\% (95\% CI $57.8,68.7)$, and $39.2 \%(95 \%$ CI $33.7,44.7)$ for those who switched from IV to SC ABA.

Conclusion. These findings support SC ABA as a well-tolerated and efficacious longterm treatment for patients with RA and inadequate response to MTX (ClinicalTrials gov identifier NCT00559585). (First Release March 1 2014; J Rheumatol 2014;41:629-39; doi:10.3899/jrheum.130112)

Key Indexing Terms:

ABATACEPT ACQUIRE RHEUMATOID ARTHRITIS LONGTERM SAFETY

From Stanford University, Palo Alto, California, USA; Universidad Autónoma de Chihuahua, Chihuahua; Unidad Reumatologica Las Americas S.C.P., Mérida, Mexico; Instituto De Ginecologia Y

Reproduccion, Lima, Peru; Organización Médica de Investigación, Buenos Aires, Argentina; Pontificial Catholic University School of Medicine, Porto Alegre, Brazil; Physician Research Collaboration, Lincoln, Nebraska, USA; University of Queensland, Brisbane, Queensland, Australia; Centro De Especialidades Médicas/Universidad Marista, Mérida, Mexico; Box Arthritis and Rheumatology of the Carolinas, Charlotte, North Carolina; Low Country Rheumatology, Charleston, South Carolina, USA; Institute of Rheumatology, Moscow, Russia; Service et Pôle de Rhumatologie, Cliniques Universitaires Saint-Luc, Institut de Recherche Expérimentale et Clinique, Université catholique de Louvain, Brussels, Belgium; Bristol-Myers Squibb, Princeton, New Jersey, USA; and Schlosspark-Klinik, Teaching Hospital of the Charite University Medicine Berlin, Berlin, Germany.

Supported by Bristol-Myers Squibb.

M.C. Genovese, MD, Stanford University; C. Pacheco Tena, MD, MSc, Universidad Autónoma de Chihuahua; A. Covarrubias, MD, Unidad

\begin{abstract}
Reumatologica Las Americas S.C.P.; G. Leon, MD, Instituto De Ginecologia Y Reproduccion; E. Mysler, MD, Organización Médica de Investigación; M. Keiserman, MD, Pontificial Catholic University School of Medicine; $R$. Valente, MD, Physician Research Collaboration; P. Nash, $M B B S, F R A C P$, University of Queensland; J.A. Simon-Campos, MD, Centro De Especialidades Médicas/Universidad Marista; J. Box, MD, Box Arthritis and Rheumatology of the Carolinas; C.W. Legerton III, MD, Low Country Rheumatology; E. Nasonov, MD, Institute of Rheumatology; P. Durez, MD, Service et Pôle de Rhumatologie, Cliniques Universitaires Saint-Luc, Institut de Recherche Expérimentale et Clinique, Université catholique de Louvain; I. Delaet, MD; Julie Teng, MD, Bristol-Myers Squibb; and R. Alten, MD, Schlosspark-Klinik, Teaching Hospital of the Charité University Medicine Berlin.

Address correspondence to Dr. M.C. Genovese, Rheumatology, 1000 Welch Road \#203, Stanford University, Palo Alto, California, USA. E-mail: genovese@stanford.edu

Full Release Article. For details see Reprints/Permissions at jrheum.org Accepted for publication December 13, 2013.
\end{abstract}

Personal non-commercial use only. The Journal of Rheumatology Copyright $\odot$ 2014. All rights reserved. 
The longterm safety and efficacy profile of intravenous (IV) abatacept (ABA), a selective $\mathrm{T}$ cell costimulation modulator, is well established in patients with rheumatoid arthritis (RA), including methotrexate (MTX)-naive patients ${ }^{1}$ and those with an inadequate response to MTX $^{2,3,4,5}$ or anti-tumor necrosis factor (TNF) therapy ${ }^{6}$. A subcutaneous (SC) formulation of ABA is also available. Four phase II or III trials have investigated the tolerability and clinical effectiveness of a weekly fixed dose of $125 \mathrm{mg}$ SC ABA, demonstrating low immunogenicity with safety and efficacy similar to that of the IV formulation ${ }^{7,8,9,10,11}$.

The Abatacept Comparison of sub(QU)cutaneous versus intravenous in Inadequate Responders to methotrexatE (ACQUIRE) trial was a multinational, phase IIIb, randomized, double-blind (DB) study that evaluated the comparable efficacy and safety of SC and IV ABA over 6 months ${ }^{12}$. At Month 6, similar proportions of SC and IV ABA-treated patients achieved an American College of Rheumatology (ACR) 20 response (estimated difference: $0.3 \%, 95 \% \mathrm{CI}-4.2,4.8)$, confirming noninferiority of SC to IV ABA. The onset and magnitude of efficacy responses were equal for both formulations, and similar patient retention was also reported (94.2\% for SC ABA vs $93.8 \%$ for IV ABA at Month 6). Overall safety was also similar between groups, including discontinuations due to adverse events (AE) and serious AE (SAE), serious infections, malignancies, and autoimmune events.

Here we describe the results from the open-label, longterm extension (LTE) of the ACQUIRE trial, in which all patients who completed the 6-month DB period received SC ABA $125 \mathrm{mg}$ weekly for up to $\sim 3.5$ years of exposure, to assess the longterm safety, efficacy, and tolerability of SC ABA.

\section{MATERIALS AND METHODS}

Study design and patient population. Patients eligible for inclusion in the open-label LTE of the ACQUIRE trial (ClinicalTrials.gov identifier NCT00559585) were required to have met the inclusion criteria for, and to have completed, the 6-month, randomized, DB period ${ }^{12}$. Patients included in the DB trial were $\geq 18$ years of age with active RA that had responded inadequately to $\geq 3$ months of treatment with MTX. Disease activity requirements at randomization were $\geq 10$ swollen and $\geq 12$ tender joints, and C-reactive protein (CRP) levels were $\geq 0.8 \mathrm{mg} / \mathrm{dl}$. Patients were screened for tuberculosis (TB) and excluded if there was current clinical/radiographic/laboratory evidence of active TB or a history of active TB in the previous 3 years. Patients with a history of active TB $\geq 3$ years ago were included if appropriately treated.

In the DB period, patients were randomized (1:1), with stratification by body weight ( $<60 \mathrm{~kg}, 60-100 \mathrm{~kg},>100 \mathrm{~kg}$ ), to receive ABA through one of 2 different routes: either SC injection $(125 \mathrm{mg})$ on Day 1 and weekly thereafter, including an IV ABA infusion $(\sim 10 \mathrm{mg} / \mathrm{kg}$ based on weight range) on Day 1 only (the SC injection was administered $\sim 30$ min after the end of the IV infusion); or IV infusion $(\sim 10 \mathrm{mg} / \mathrm{kg}$ based on weight range) on Days 1, 15, and 29, and every 4 weeks thereafter (up to Day 162 for the $\mathrm{SC}$ ABA group, and up to Day 141 for the IV ABA group). During the open-label LTE, all eligible patients received SC ABA $125 \mathrm{mg}$ weekly (from Day 169 of the DB period/Day 1 of the LTE).

During the DB period, MTX was maintained at the same dose as at trial entry ( $\geq 15 \mathrm{mg} /$ week); low-dose stable oral corticosteroids ( $\leq 10 \mathrm{mg} /$ day, prednisone equivalent) and stable-dose nonsteroidal antiinflammatory drugs (NSAID) were also permitted. Adjustments to MTX, corticosteroids, and NSAID were permitted during the LTE at the discretion of the investigator, based on the clinical status of the patient. During the LTE period, sites were allowed to add traditional disease-modifying antirheumatic drugs (DMARD) at the discretion of the investigator. The addition of other biologic agents was not permitted.

Assessments. Safety assessments were performed at each study visit during the DB period, and at 12-week intervals and yearly visits during the LTE. All AE, SAE, AE of interest, vital signs, and laboratory test abnormalities were recorded. AE of interest included infections, malignancies, autoimmune disorders, and injection-site reactions. Immunogenicity was evaluated by ELISA during the DB period of this study ${ }^{12}$. During the LTE, however, a different assay was used to assess immunogenicity - electrochemiluminescence (ECL), based on the proportion of patients with a seropositive response defined as a titer value of $\geq 10$. The ECL immunoassay detected antibodies to CTLA-4 and possibly immunoglobulin (Ig), or the $\mathrm{IgG}$ and/or junction region. Persistent immunogenicity was defined as detection of at least 2 consecutive positive on-treatment serum samples within the same antibody reactivity. Efficacy assessments were performed at baseline and at each study visit during the DB period, at 12-week intervals and yearly visits during the LTE, and 7 days after the last SC injection for patients who discontinued the study prematurely. Improvement in signs and symptoms of RA was evaluated by ACR 20, 50, and 70 responses. Disease activity was evaluated by 28 -joint Disease Activity Score (DAS28) based on CRP, Clinical Disease Activity Index (CDAI), and Simplified Disease Activity Index (SDAI) scores. The Health Assessment Questionnaire-Disability Index (HAQ-DI) was used to assess physical function, with a HAQ-DI response defined as an improvement from baseline of $\geq 0.3$ units.

Statistical analyses. Safety assessments are presented for all patients who received at least 1 dose of SC ABA during the LTE; data are presented up to a maximum of 44 months of exposure following study initiation (which corresponds to the database cutoff date), and include events that occurred up to Day 56 (greater than 4 times the half-life of ABA in patients with RA) after the last dose of study drug for patients who discontinued the LTE. Safety data from the DB period are described according to original DB treatment group, to evaluate whether there is an increase in the incidence of safety events over time; DB data are presented up to the first dose of study drug in the LTE, or up to 56 days after the last dose of study drug for patients who discontinued the DB period or did not enter the LTE. Results from the safety assessments are presented as frequencies and incidence rates (IR). IR were calculated as events per 100 patient-years of exposure, with a patient's contribution to exposure ending at the time of the first occurrence of an AE.

Efficacy data are based on as-observed analyses, including all patients who entered the LTE and received at least 1 dose of ABA, and are presented according to patients' original DB treatment group (SC or IV ABA). Data are presented up to $\sim 33$ months (Day 981) of the study. Although the maximum time of exposure to $\mathrm{ABA}$ during the trial was 44 months, most patients had not reached this timepoint at time of analysis because of differential enrollment dates. Most patients had, however, reached Day 981 at the time of reporting, and we report efficacy up to this timepoint. No formal statistics were performed during the LTE, but descriptive statistics are provided for all assessments, including point estimates and 95\% CI for the proportions of patients achieving each clinical efficacy outcome.

\section{RESULTS}

Patient disposition and baseline clinical characteristics. A total of 1372 patients continued into the LTE, accounting for $99.1 \%$ of patients who completed the 6-month DB period (Figure 1). At the time of reporting (as of October 3, 2011, 


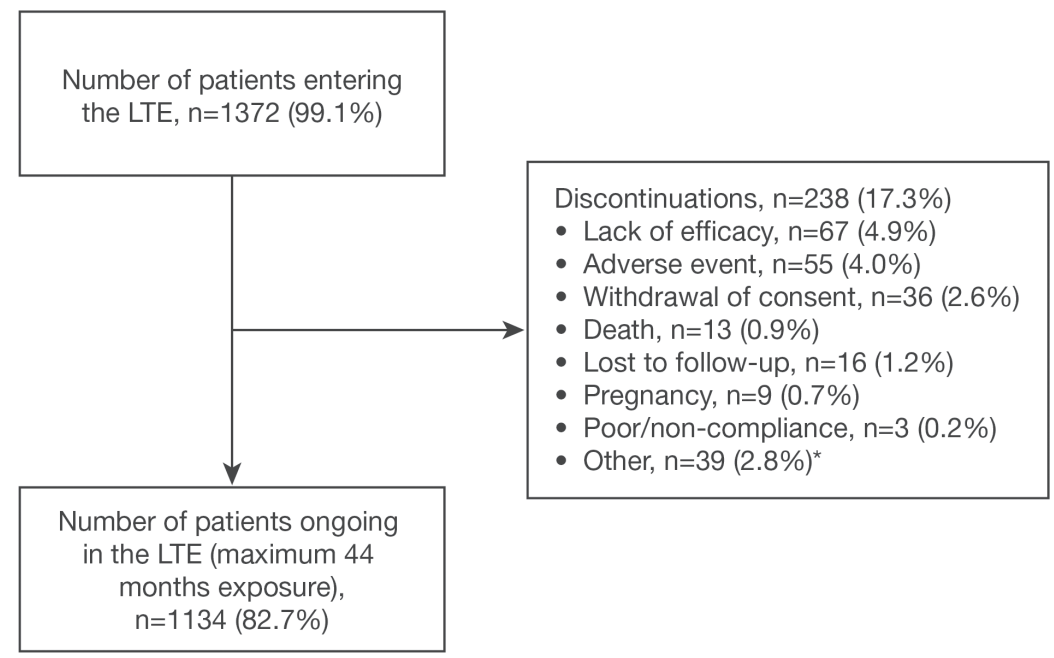

Figure 1. Patient disposition in the longterm extension (LTE). *Includes "Other", "Administrative reason by sponsor", and "Subject no longer meets study criteria" discontinuation categories. Last timepoint for the LTE is October 3, 2011, at which point mean (range) cumulative duration of abatacept exposure was $31.9(8-44)$ months.

database lock, which corresponds to a maximum possible exposure time of 44 months), 1134 (82.7\%) patients remained in the study and continued to receive SC ABA. During the LTE, $4.9 \%(\mathrm{n}=67)$ of patients discontinued owing to lack of efficacy, and $4.0 \%(n=55)$ discontinued because of an AE. Mean (SD) duration of ABA exposure during the cumulative study period (DB plus LTE periods) was 31.9 (6.8) months; the median exposure was 33.0 (range $8-44)$ months. As the wide range of exposure indicates, some patients had not yet reached the later assessment timepoints at the time of the efficacy analysis presented here because of differential enrollment dates; this is reflected in decreased patient numbers at these later timepoints.

Demographic and clinical characteristics at baseline for patients treated in the LTE were similar to those observed for the intent-to-treat population of patients treated in the DB period (Table 1) ${ }^{12}$. Patients had longstanding [mean 7.6 (7.9) yrs], moderate-to-severe disease, as evidenced by tender and swollen joint counts of 29.6 (13.8) and 19.9 (9.2), respectively, and a mean DAS28-CRP of $6.2(0.9)$.

Table 1. Baseline demographic and clinical characteristics for patients treated in the double-blind (DB) period and longterm extension (LTE). Data are mean (SD), unless otherwise stated.

\begin{tabular}{|c|c|c|c|}
\hline \multirow[b]{2}{*}{ Characteristics } & \multicolumn{2}{|c|}{ DB Population } & \multirow{2}{*}{$\begin{array}{l}\text { LTE Population } \\
\text { SC ABA + MTX, } \\
\quad \mathrm{n}=1372\end{array}$} \\
\hline & $\begin{array}{c}\mathrm{SC} \text { ABA + MTX } \\
\mathrm{n}=736\end{array}$ & $\begin{array}{c}\text { IV ABA + MTX, } \\
n=721\end{array}$ & \\
\hline Age, yrs & $49.9(13.2)$ & $50.1(12.6)$ & $49.7(12.8)$ \\
\hline Sex, female $\%$ & 84.4 & 80.4 & 82.4 \\
\hline Race, white $\%$ & 74.7 & 74.5 & 74.5 \\
\hline Disease duration, yrs & $7.6(8.1)$ & $7.7(7.8)$ & $7.6(7.9)$ \\
\hline Tender joint count & $30.1(14.1)^{*}$ & $29.1(13.3)$ & $29.6(13.8)^{5}$ \\
\hline Swollen joint count & $20.4(9.6)^{\dagger}$ & $19.4(8.6)$ & $19.9(9.2)^{9}$ \\
\hline DAS28-CRP & $6.2(0.9)^{\S}$ & $6.2(0.8)$ & $6.2(0.9)^{* *}$ \\
\hline $\mathrm{CRP}, \mathrm{mg} / \mathrm{dl}$ & $2.6(2.9)^{\ddagger}$ & $2.7(2.9)$ & $2.7(3.0)^{9}$ \\
\hline HAQ-DI score & $1.7(0.7)$ & $1.7(0.7)$ & $1.7(0.7)$ \\
\hline Pain, 100 mm VAS & $67.8(20.1)$ & $66.8(20.5)$ & $67.2(20.3)$ \\
\hline Patient global assessment, $100 \mathrm{~mm}$ VAS & $66.8(20.4)$ & $64.9(20.0)$ & $65.9(20.2)$ \\
\hline Physician global assessment, $100 \mathrm{~mm}$ VAS & $64.3(16.5)$ & $63.1(16.6)$ & $63.6(16.4)$ \\
\hline RF-positive \% & $84.8^{\ddagger}$ & $85.9^{11}$ & $86.1^{\dagger \dagger}$ \\
\hline
\end{tabular}

$*^{*} \mathrm{n}=736 .{ }^{\dagger} \mathrm{n}=735 .{ }^{\dagger} \mathrm{n}=734 .{ }^{\S_{n}} \mathrm{n}=733 .{ }^{11} \mathrm{n}=711 .{ }^{9} \mathrm{n}=1371 . * * \mathrm{n}=1370 .{ }^{\dagger \dagger} \mathrm{n}=1350$. CRP: C-reactive protein; DAS28: 28-joint Disease Activity Score; HAQ-DI: Health Assessment Questionnaire-Disability Index; IV: intravenous; RF: rheumatoid factor; SC: subcutaneous; VAS: visual analog scale; ABA: abatacept; MTX: methotrexate. 
Concomitant medications. Nearly all patients (99.6\%) continued to receive MTX, and mean MTX dose remained consistent throughout the LTE of the ACQUIRE study, during which time adjustments were permitted. Weekly MTX doses were 16.2 (4.8), 15.9 (6.0), 15.8 (6.7), 15.7 (7.4), and 15.5 (8.4) at Days 169, 365, 533, 729, and 897, respectively. At randomization, $69.2 \%$ of patients were receiving oral steroids, at a mean (SD) dose of $4.6 \mathrm{mg}$ (3.7); the proportions of patients receiving oral steroid remained relatively consistent throughout the trial, with $72.7 \%$ of patients receiving oral steroids at some point during the LTE, at a dose of $5.1 \mathrm{mg}$ (5.4). During the LTE, 41 patients (3.0\%) added hydroxychloroquine, 36 (2.6\%) added sulfasalazine, and $<1 \%$ added leflunomide, chloroquine, cyclosporine, azathioprine, or gold sodium.

Safety. The overall safety experience, assessed by the IR of events, in the LTE (maximum of $\sim 3$ yrs' exposure) was similar to the experience reported in the 6-month DB period with both SC and IV ABA (Table 2). The IR of SAE was 8.76 (events per 100 patient-yrs; 95\% CI 7.71, 9.95) during the LTE, which is comparable to that observed among patients receiving SC ABA in the DB period $(9.02,95 \%$ CI $6.31,12.90)$. SAE resulted in discontinuation in 42 patients (3.1\%) during the LTE, with the most common events (occurring in more than 1 patient) being breast cancer (5 patients, $0.4 \%$ ), malignant lung neoplasm ( 3 patients, $0.2 \%$ ), pneumonia, pulmonary tuberculosis and myocardial infarction (MI; 2 patients each, $0.1 \%$ ). Twenty-one deaths $(1.5 \%)$ were reported during the LTE, at an IR of $0.71(95 \%$ CI $0.44,1.09)$. These included sudden death, death with cause not specified, asthma attack, car accident, cardiac arrest, acute gastrointestinal infection, peritoneal carcinomatosis, multiple organ failure, secondary pneumonia and pulmonary TB, lung cancer, $\mathrm{C} 1$ fracture, primary lung cancer, pneumonia, acute MI, anal cancer, bronchopneumonia and coronary heart failure, sudden circulatory arrest, sudden cardiac death, metastatic cholangiocarcinoma, and MI.
Infections occurred at an IR of 44.80 (95\% CI 41.81, $48.01)$ and serious infections occurred at an IR of $1.72(95 \%$ CI $1.30,2.27)$ during the LTE period, and were not increased compared with the 6-month DB period (Table 3). Serious infections occurring in more than 1 patient each during the LTE included pneumonia (10 patients, $0.7 \%$, IR $0.34,95 \%$ CI $0.18,0.63$ ); bronchitis and urinary tract infection (4 patients each, 0.3\%, IR 0.14, 95\% CI 0.05 , $0.36)$; appendicitis and herpes zoster ( 3 patients each: $0.2 \%$, IR $0.10,95 \%$ CI 0.03, 0.31; and 0.10, 95\% CI 0.03, 0.32, respectively); and gastroenteritis, lobar pneumonia, pulmonary $\mathrm{TB}$, acute pyelonephritis, respiratory tract infection and sinusitis ( 2 patients each, $0.1 \%$, IR $0.07,95 \%$ CI $0.02,0.27)$. Seven opportunistic infections occurred during the study, all during the LTE, including TB in 4 patients (IR 0.14, 95\% CI 0.05, 0.36) and candidiasis in 3 patients (IR $0.10,95 \%$ CI $0.03,0.31$ ). The patients with TB were a 35-year-old woman from South America with normal chest radiograph and negative purified protein derivative (PPD) at baseline, who had a positive culture on Day 349, was diagnosed with Grade II TB and discontinued study drug; a 56-year-old woman from Asia who had a normal chest radiograph and negative PPD test at baseline, developed pulmonary TB on the left lung based on PPD, quantiferon, chest radiograph, and computed tomography scan results at Day 317 and dropped out of the study; a 55-year-old man from South America who had a history of $\mathrm{TB}$ in childhood (treated with unknown antituberculous therapy for $1 \mathrm{yr}$ ), diagnosed with peritoneal TB on Day 537 of the study and subsequently discontinued; a 64-year-old woman from South America with no history of TB who developed secondary pneumonia and pulmonary TB on Day 791 of treatment and discontinued the study drug.

The IR of malignancies did not increase during the LTE period $(1.19 ; 95 \%$ CI $0.86,1.66)$ compared with the DB period (Table 2). Events that occurred in more than 1 patient included basal cell carcinoma ( 9 patients, $0.7 \%$, IR 0.31 ;

Table 2. Summary of adverse events reported in the double-blind (DB) period and longterm extension (LTE). Table includes data from patients included in the anti-tumor necrosis factor failure substudy. Safety data are based on all patients who received at least 1 dose of abatacept (ABA) in the intent-to-treat population.

\begin{tabular}{|c|c|c|c|c|c|c|}
\hline Patie & $\begin{array}{l}\text { ents with Eve } \\
\text { n (\%) }\end{array}$ & IR $(95 \% \mathrm{CI})$ & $\begin{array}{c}\text { Patients with Event, } \\
\mathrm{n}(\%)\end{array}$ & IR $(95 \% \mathrm{CI})$ & $\begin{array}{c}\text { Patients with Event, } \\
\mathrm{n}(\%)\end{array}$ & IR $(95 \% \mathrm{CI})$ \\
\hline SAE & $31(4.2)$ & $9.02(6.31,12.90)$ & $36(4.9)$ & $11.14(8.04,15.44)$ & $238(17.3)$ & $8.76(7.71,9.95)$ \\
\hline Discontinued owing to SAE & $9(1.2)$ & $2.66(1.22,5.06)$ & $14(1.9)$ & $4.25(2.32,7.13)$ & $42(3.1)$ & $1.42(1.03,1.92)$ \\
\hline $\mathrm{AE}$ & $503(67.6)$ & $279.35(255.46,304.86)$ & $478(65.4)$ & $265.67(242.38,290.59)$ & 9) $1147(83.6)$ & $107.73(101.59,114.15)$ \\
\hline Serious infections & $5(0.7)$ & $1.48(0.62,3.56)$ & $10(1.4)$ & $3.05(1.64,5.67)$ & $50(3.6)$ & $1.72(1.30,2.27)$ \\
\hline Malignancies & $2(0.3)$ & $0.59(0.15,2.36)$ & $5(0.7)$ & $1.52(0.63,3.65)$ & $35(2.6)$ & $1.19(0.86,1.66)$ \\
\hline Autoimmune events & $6(0.8)$ & $1.78(0.80,3.96)$ & $6(0.8)$ & $1.83(0.82,4.07)$ & $38(2.8)$ & $1.31(0.95,1.79)$ \\
\hline
\end{tabular}

AE: adverse event; IV: intravenous; MTX: methotrexate; SAE: serious adverse event; SC: subcutaneous; IR: incidence rates. Personal non-commercial use only. The Journal of Rheumatology Copyright @ 2014 . All rights reserved. 
Table 3. Subcutaneous injection-site reactions reported in the double-blind (DB) period and longterm extension (LTE). Data are n (\%) patients with event.

\begin{tabular}{|c|c|c|c|}
\hline Reaction Type & $\begin{array}{c}\text { DB Period SC ABA + MTX, } \\
n=736\end{array}$ & $\begin{array}{c}\text { DB Period IV ABA + MTX, } \\
n=721\end{array}$ & $\begin{array}{c}\text { LTE SC ABA + MTX, } \\
n=1372\end{array}$ \\
\hline Subcutaneous injection-site reaction & $19(2.6)$ & $19(2.6)$ & $27(2.0)$ \\
\hline Hematoma & $4(0.5)$ & $5(0.7)$ & $6(0.4)$ \\
\hline Pain & $1(0.1)$ & $4(0.5)$ & $6(0.4)$ \\
\hline Unspecified reaction & $1(0.1)$ & $3(0.4)$ & $4(0.3)$ \\
\hline Papule & $1(0.1)$ & $3(0.4)$ & $2(0.1)$ \\
\hline Rash & $2(0.3)$ & $1(0.1)$ & $1(<0.1)$ \\
\hline Other* & $4(0.5)$ & 0 & $4(0.3)$ \\
\hline
\end{tabular}

*Other injection-site reactions reported in only 1 patient each included inflammation, nodule, paresthesia, and swelling. Safety data are based on all patients who received at least 1 dose of abatacept in the intent-to-treat population. IV: intravenous; ABA: abatacept; MTX: methotrexate; SC: subcutaneous.

$95 \%$ CI $0.16,0.59)$, breast cancer ( 5 patients, $0.4 \%$, IR 0.17 ; 95\% CI $0.07,0.41$ ), malignant lung neoplasm (3 patients, $0.2 \%$, IR $0.10 ; 95 \%$ CI $0.03,0.31$ ), squamous cell carcinoma of skin (3 patients, $0.2 \%$, IR 0.10 ; $95 \%$ CI 0.03 , 0.31 ), and prostate cancer ( 2 patients, $0.1 \%$, IR $0.07 ; 95 \%$ CI $0.02,0.27$ ).

Autoimmune events were reported at an IR of $1.31(95 \%$ CI $0.95,1.79)$ during the LTE, and were not increased relative to the DB period (Table 2). The most frequently reported events were psoriasis ( 9 patients, $0.7 \%$, IR 0.31 ; $95 \%$ CI $0.16,0.59$ ), of which 2 events were exacerbations in patients previously diagnosed, and Sjögren syndrome (7 patients, $0.5 \%$, IR $0.24 ; 95 \%$ CI $0.11,0.50$ ), one of which was classified as secondary, followed by episcleritis, vasculitis, erythema nodosum, and ulcerative colitis, which occurred in 3 patients each $(0.2 \%$, IR $0.10 ; 95 \%$ CI 0.03 , 0.31 ). Autoimmune events in the LTE required interruption of treatment in 3 cases (Crohn disease, erythema nodosum, and psoriasis, 1 patient each) and discontinuation of treatment in 2 patients (leukocytoclastic vasculitis and multiple sclerosis).

During the LTE, 27 patients (2.0\%) experienced a local injection-site reaction. All events were mild, except for a single event of moderate intensity (injection-site nodule). Other events included 6 erythema $(0.4 \%), 6$ hematoma $(0.4 \%), 6$ pain $(0.4 \%), 4$ unspecified reaction $(0.3 \%), 3$ pruritus $(0.2 \%), 2$ hemorrhage $(0.1 \%), 2$ papule $(0.1 \%)$, and 1 rash $(<0.1 \%)$. The frequency of injection-site reactions with SC ABA during the LTE was comparable with the frequency reported for SC ABA and IV ABA (SC placebo) in the DB period [19 (2.6\%) and 19 (2.6\%), respectively].

Safety over time. The IR of SAE at 6-month intervals did not increase over time compared with that during the DB period (Figure 2). Similarly, over time, the IR by 6-month intervals for serious infections, malignancies, and autoimmune disorders did not increase with increasing ABA exposure (Figure 2). Note that at the final timepoint, $95 \%$ CI for SAE and serious infections are wide owing to diminishing patient numbers.

Immunogenicity. During the LTE, a total of $142 / 1372$ patients $(10.3 \%)$ had a laboratory-reported positive antibody response to ABA. Only 29/1350 patients (2.1\%) experienced persistent immunogenicity (defined as at least 2 consecutive positive samples); 23 patients (1.7\%) had a persistent response while taking treatment; and 7 patients $(0.5 \%)$ had a persistent response while not taking treatment. Among those patients who had a persistent response while taking treatment, $11(0.8 \%)$ patients had a response to CTLA-4 and possibly Ig, and 12 patients (0.9\%) had a response to Ig and/or the junction region. The majority of patients had low titers; $7 / 142$ patients $(4.9 \%)$ had at least 1 titer sample $\geq 100$ and $0 / 142$ patients $(0 \%)$ experienced persistent high titers (defined as at least 2 consecutive samples $\geq 100$ ).

No associations between immunogenicity and safety were observed. Among patients who experienced a positive antibody response to ABA, SAE were reported in 19/142 patients (13.4\%), which was consistent with the incidence in the overall population (Table 2). The percentage of patients with a positive immunogenicity response and infections [88/142 (62.0\%)], serious infections [3/142 (2.1\%)], malignancies [2/142(1.4\%)], autoimmune events [4/142 (2.8\%)], and local injection-site reactions $[1 / 142(0.7 \%)]$ was also consistent with the overall LTE population (Table 2).

The efficacy of SC ABA in patients with positive antibody responses was consistent with the results of the overall population reported below. Among patients who experienced a positive immunogenicity response and were taking treatment on Day $981(\mathrm{n}=53)$, ACR 20, 50, and 70 response rates were $86.8 \%$ (95\% CI 77.7, 95.9), 67.9\% (95\% CI 55.4, 80.5), and 34.0\% (95\% CI 21.2, 46.7), respectively.

Efficacy. During the original DB period, comparable clinical and functional efficacy was observed through Day 169 (end

Personal non-commercial use only. The Journal of Rheumatology Copyright ( 2014. All rights reserved. 

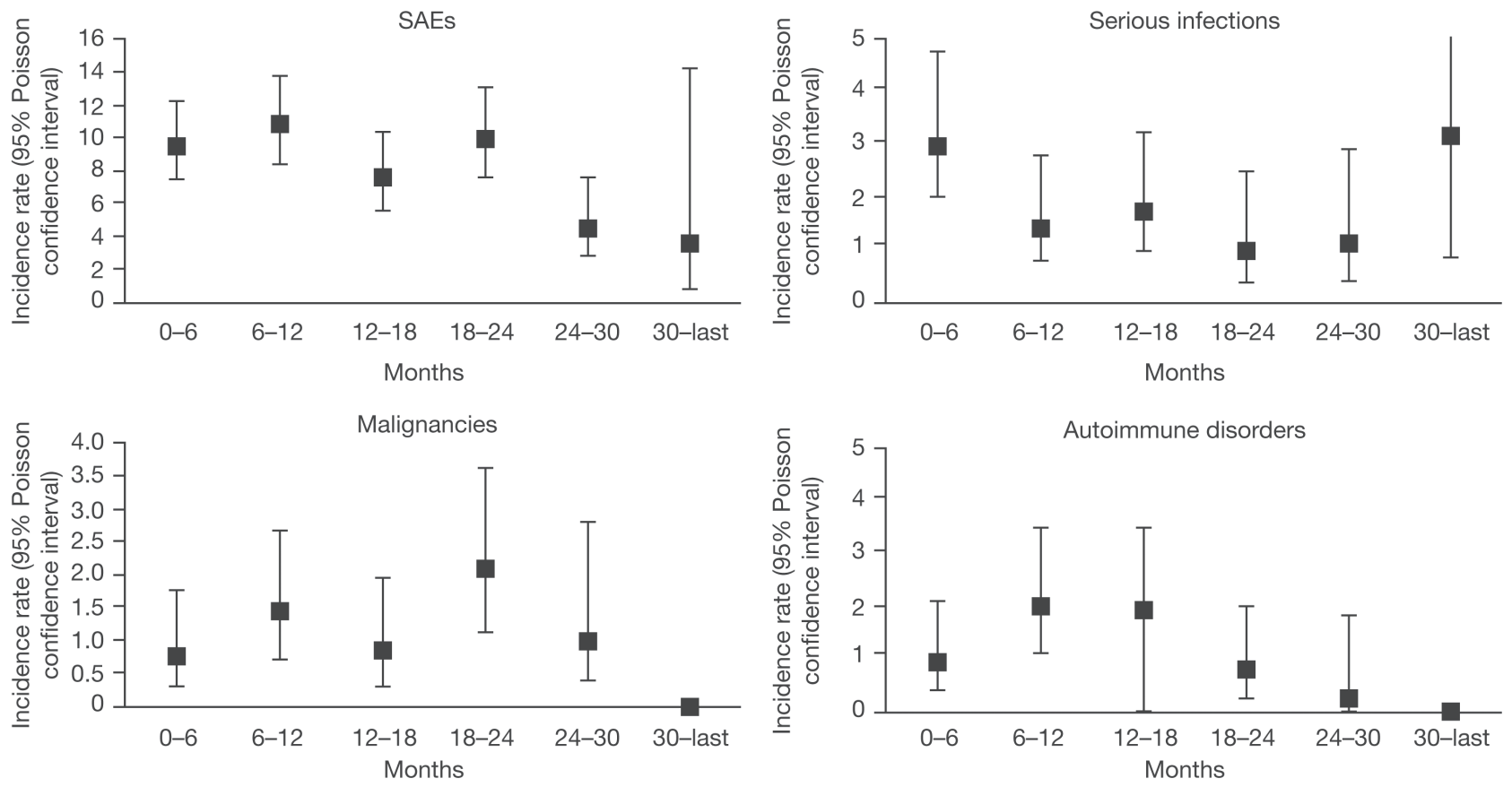

\begin{tabular}{|l|l|c|c|c|c|c|c|}
\hline Months (days) & & $0-6(1-180)$ & $6-12$ (181-360) & 12-18 (361-540) & 18-24 (541-720) & 24-30 (721-900) & 30-Last (901-Last) \\
\hline Total & SAEs/serious infections & 658.57 & 603.74 & 552.13 & 514.86 & 331.36 & 56.45 \\
exposure & Malignancies & 668.90 & 638.77 & 604.21 & 579.03 & 379.11 & 63.81 \\
(person years) & Autoimmune events & 668.36 & 636.21 & 597.13 & 569.46 & 374.58 & 64.30 \\
\hline
\end{tabular}

Figure 2. Incidence rates of adverse events (AE) by 6-month intervals for patients treated in the longterm extension. Includes data up to 56 days after the last dose of study drug. For patient-year calculation, patients who reported an AE in a previous 6-month interval did not contribute to total exposure in the next 6-month interval (even when those patients had exposure information in the next 6-month interval). SAE: serious adverse event; py: patient-year.

of $\mathrm{DB}$ period) for the original SC and IV ABA treatment groups. During the LTE, the original IV ABA arm demonstrated continuous clinical and functional improvement following the switch to SC ABA, comparable to the continuous clinical and functional improvement seen in patients from the original SC ABA arm (Figures 3 and 4). Although most patients reached Day 981 by the time of this analysis, not all patients had reached this point as a result of different enrollment dates in the trial. This resulted in lower patient numbers at the later time points of the efficacy analyses.

For the original SC ABA and IV ABA groups, respectively, ACR responses were maintained from Day 169 through Day 981 (Figure 3A). On Day 169, ACR 20 response rates were $80.2 \%$ (95\% CI 77.2, 83.2) and $80.0 \%$ (95\% CI 77.0, 83.0); ACR 50 response rates were $53.4 \%$ (95\% CI 49.7, 57.1) and 52.8\% (95\% CI 49.1, 56.6); and ACR 70 response rates were $27.7 \%(95 \%$ CI $24.3,31.0)$ and 26.7\% (95\% CI 23.3, 30.0). On Day 981, ACR 20 response rates were $84.8 \%(95 \% \mathrm{CI} 80.8,88.8)$ and $84.7 \%(95 \% \mathrm{CI}$ 80.7, 88.8); ACR 50 response rates were $63.5 \%$ (95\% CI $58.2,68.9)$ and $63.2 \%$ (95\% CI 57.8, 68.7); and ACR 70 response rates were $39.5 \%$ (95\% CI 34.0, 44.9) and 39.2\% (95\% CI 33.7, 44.7).

Improvements in physical function outcomes seen during the DB period were also maintained throughout the LTE for both the original SC and IV ABA groups, respectively (Figure 3B). On Day 169, 72.6\% (95\% CI 69.3, 76.0) and $68.3 \%$ (95\% CI 64.8, 71.8); and on Day 981, 73.8\% (95\% CI 68.9, 78.7) and 70.0\% (95\% CI 64.8, 75.1) had achieved a HAQ-DI response.

Disease activity outcomes achieved in the DB period were maintained for patients who remained on treatment, for both original DB treatment groups (Figure 4). The proportions of patients achieving a DAS28-CRP $\leq 3.2$ were $39.9 \%(95 \%$ CI $36.2,43.5)$ and $41.7 \%$ (95\% CI $37.9,45.4)$ on Day 169 for the original SC and IV treatment groups, respectively; and were $55.2 \%$ (95\% CI $49.5,61.0)$ and $57.1 \%(95 \%$ CI 51.2, 62.9) on Day 981 (Figure 4A). The proportions of patients achieving DAS28-CRP $<2.6$ were $24.2 \%$ (95\% CI 21.0, 27.4) and $25.0 \%$ (95\% CI 21.7, 28.3) on Day 169 for the original $\mathrm{SC}$ and IV ABA treatment groups, respectively, and were $38.5 \%(95 \%$ CI $32.9,44.2)$ and $34.5 \%$ (95\% CI 28.9 ,

Personal non-commercial use only. The Journal of Rheumatology Copyright @ 2014 . All rights reserved. 


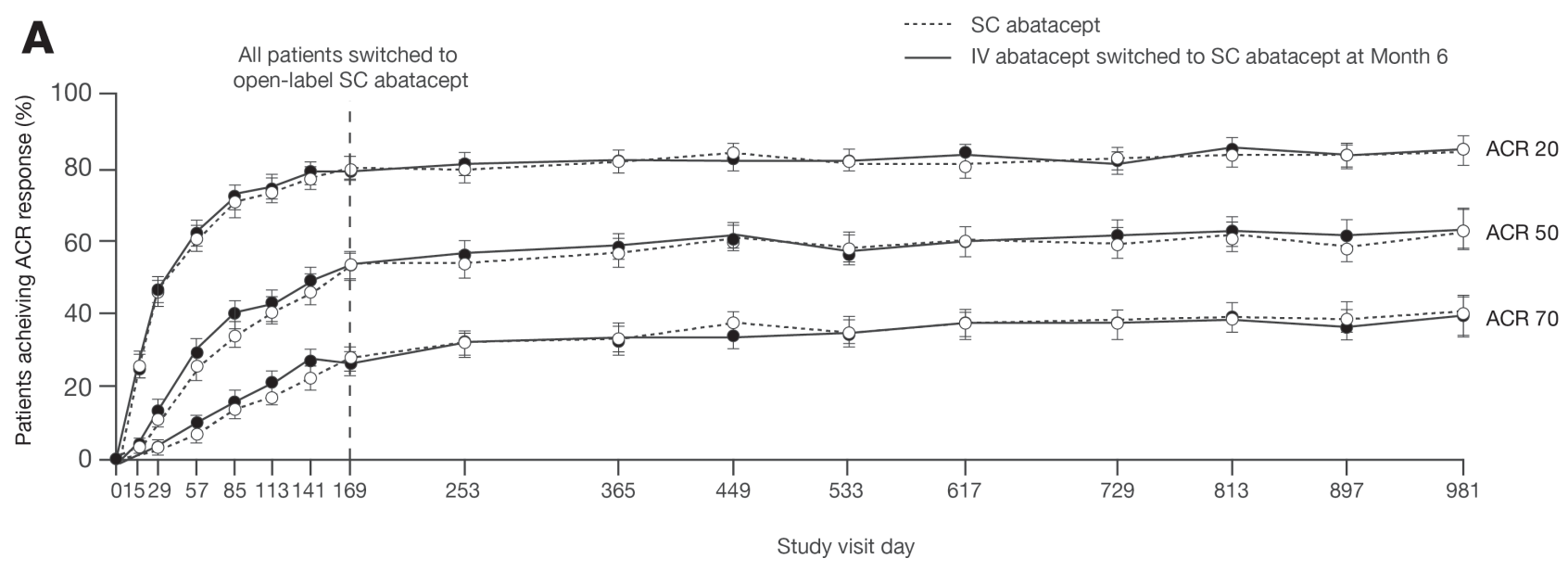

\begin{tabular}{|c|c|c|c|c|c|c|c|c|c|c|c|c|c|c|c|c|c|}
\hline & & \multicolumn{16}{|c|}{ Patients with available data at each visit day*, according to the original treatment group } \\
\hline & & 15 & 29 & 57 & 85 & 113 & 141 & 169 & 253 & 365 & 449 & 533 & 617 & 729 & 813 & 897 & 981 \\
\hline \multirow{2}{*}{ ACR 20} & abatacept & 685 & 687 & 684 & 685 & 687 & 685 & 686 & 685 & 661 & 648 & 627 & 611 & 600 & 585 & 525 & 310 \\
\hline & IV switched to SC abatacept & 665 & 668 & 668 & 669 & 670 & 671 & 670 & 667 & 652 & 635 & 620 & 606 & 587 & 573 & 515 & 301 \\
\hline \multirow{2}{*}{ ACR 50} & SC abatacept & 688 & 690 & 687 & 686 & 686 & 684 & 689 & 683 & 663 & 648 & 628 & 613 & 598 & 585 & 529 & 310 \\
\hline & IV switched to $S C$ & 669 & 673 & 671 & 672 & 670 & 671 & 672 & 668 & 653 & 635 & 620 & 606 & 587 & 573 & 517 & 302 \\
\hline \multirow{2}{*}{ ACR 70} & SC abatacept & 689 & 689 & 688 & 686 & 689 & 690 & 690 & 685 & 666 & 650 & 626 & 616 & 600 & 584 & 530 & 309 \\
\hline & IV switched to SC abatacept & 669 & 673 & 670 & 672 & 669 & 671 & 671 & 666 & 655 & 637 & 619 & 606 & 586 & 574 & 516 & 301 \\
\hline
\end{tabular}

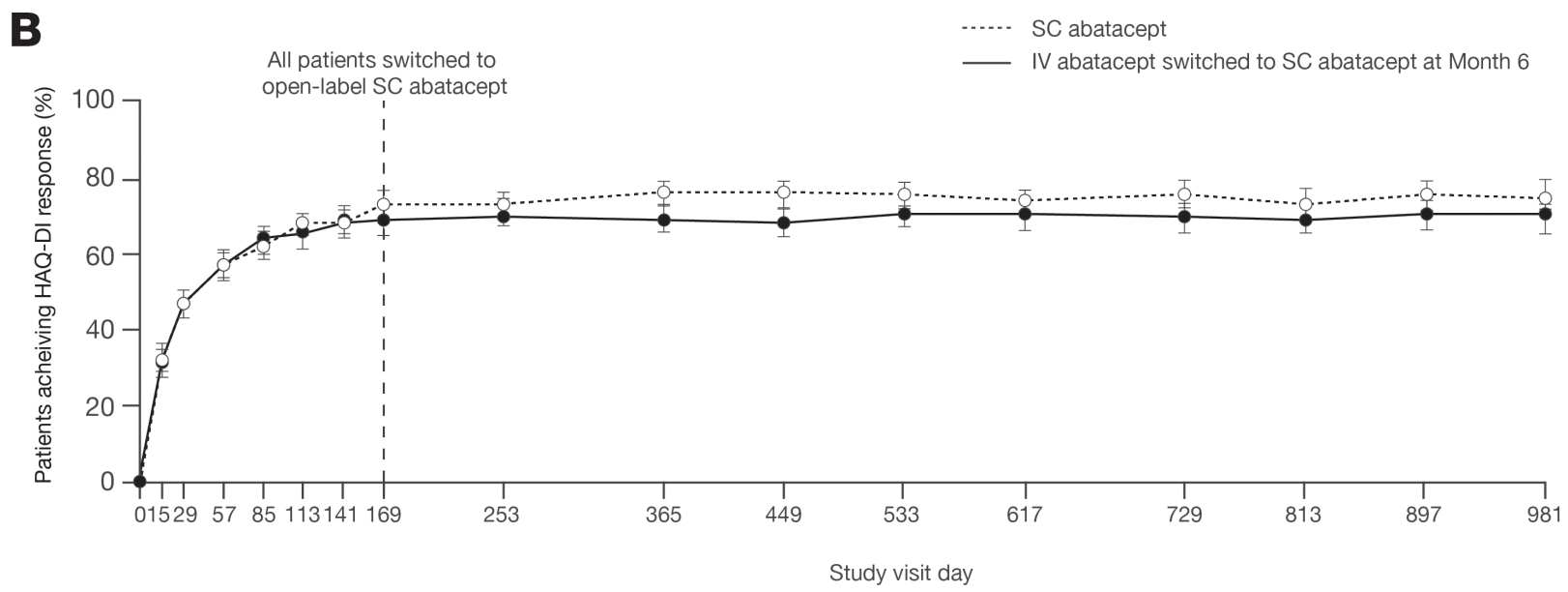

\begin{tabular}{|l|c|c|c|c|c|c|c|c|c|c|c|c|c|c|c|c|}
\hline & \multicolumn{8}{|c|}{ Patients with available data at each visit day ${ }^{*}$, according to the original treatment group } \\
\cline { 2 - 30 } & 15 & 29 & 57 & 85 & 113 & 141 & 169 & 253 & 365 & 449 & 533 & 617 & 729 & 813 & 897 & 981 \\
\hline SC abatacept & 689 & 690 & 688 & 687 & 691 & 690 & 691 & 685 & 666 & 652 & 629 & 617 & 602 & 588 & 530 & 313 \\
\hline IV switched to SC abatacept & 669 & 673 & 671 & 673 & 673 & 672 & 672 & 668 & 656 & 637 & 623 & 607 & 588 & 575 & 516 & 303 \\
\hline
\end{tabular}

Figure 3. Clinical and functional efficacy according to American College of Rheumatology responses over time (A) and HAQ-DI response rates over time (B). HAQ response is defined as an improvement of at least 0.3 units from baseline. As-observed analysis, based on patients entering the longterm extension who received at least 1 dose of abatacept. *Not all patients reached later timepoints at time of data analysis. ACR: American College of Rheumatology; IV: intravenous; SC: subcutaneous; HAQ-DI: Health Assessment Questionnaire-Disability Index.

40.2) on Day 981 (Figure 4A). For patients entering the LTE, the mean baseline CDAI score was 43.86 (12.34), and the SDAI score was 46.54 (13.11). The proportions of patients achieving CDAI-defined remission (score $\leq$
2.8) and SDAI-defined remission (score $\leq 3.3$ ) were maintained from Day 169 through Day 981 among patients entering the LTE, for both DB treatment groups (Figures 4B and 4C).

Personal non-commercial use only. The Journal of Rheumatology Copyright @ 2014 . All rights reserved. 


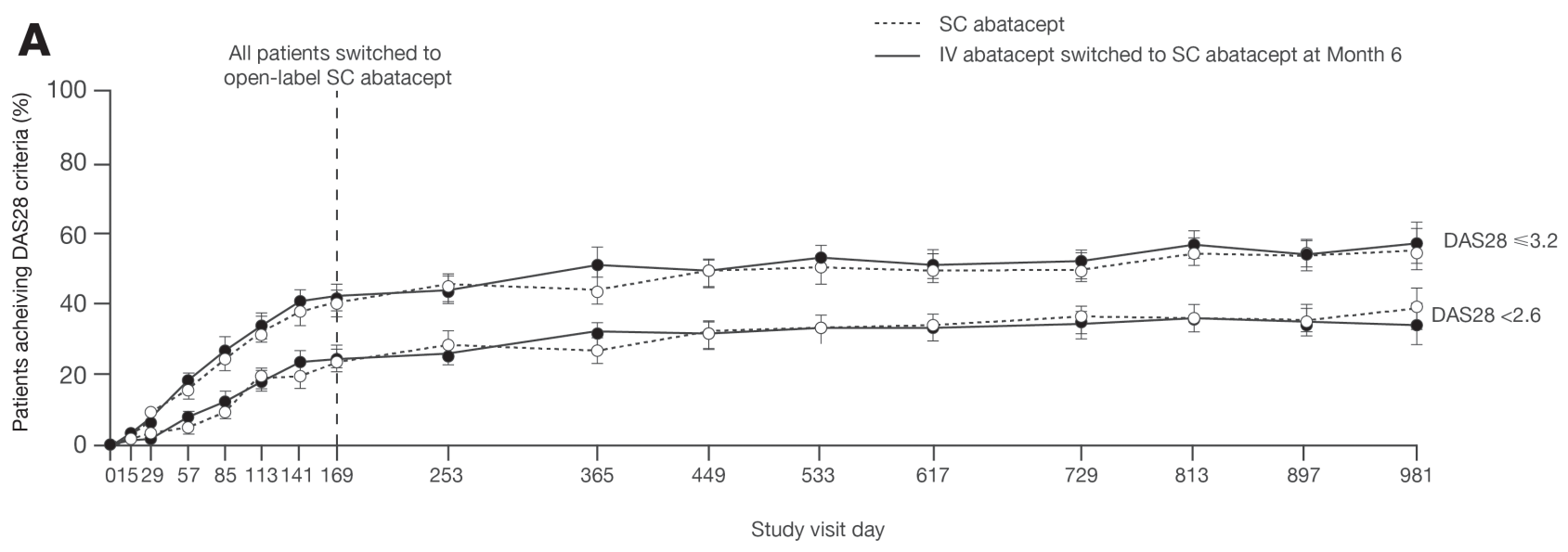

\begin{tabular}{|c|c|c|c|c|c|c|c|c|c|c|c|c|c|c|c|c|}
\hline & \multicolumn{16}{|c|}{ Patients with available data at each visit day*, according to the original treatment group } \\
\hline & 15 & 29 & 57 & 85 & 113 & 141 & 169 & 253 & 365 & 449 & 533 & 617 & 729 & 813 & 897 & 981 \\
\hline SC abatacept & 685 & 685 & 680 & 679 & 680 & 678 & 685 & 684 & 657 & 646 & 627 & 613 & 598 & 578 & 519 & 288 \\
\hline IV switched to SC abatacept & 659 & 668 & 666 & 657 & 663 & 662 & 667 & 660 & 651 & 631 & 616 & 601 & 584 & 566 & 507 & 275 \\
\hline
\end{tabular}

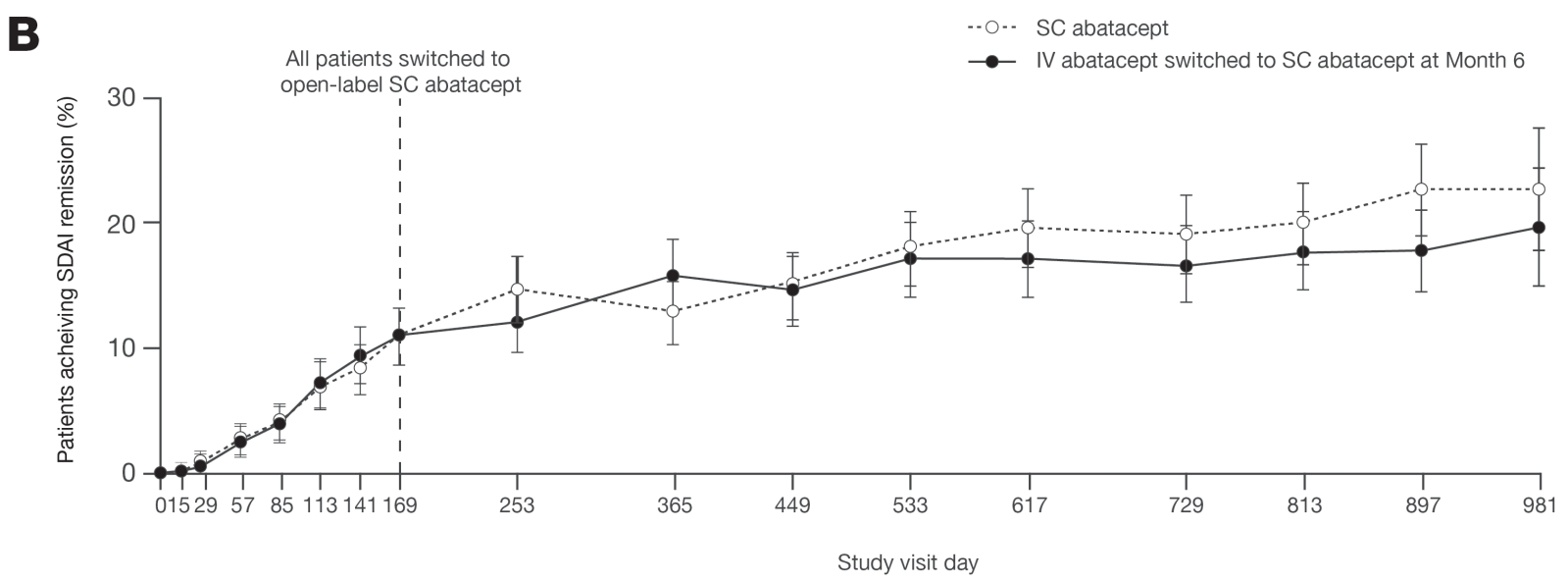

\begin{tabular}{|c|c|c|c|c|c|c|c|c|c|c|c|c|c|c|c|c|}
\hline & \multicolumn{16}{|c|}{ Patients with available data at each visit day*, according to the original treatment group } \\
\hline SC abatacept & 684 & 683 & 679 & 678 & 679 & 677 & 684 & 681 & 656 & 646 & 626 & 613 & 598 & 577 & 518 & 287 \\
\hline IV switched to SC abatacept & 656 & 665 & 665 & 656 & 662 & 662 & 665 & 659 & 651 & 630 & 615 & 601 & 583 & 566 & 506 & 275 \\
\hline
\end{tabular}

Figure 4. Disease activity outcomes according to DAS28-CRP outcomes over time (A), SDAI remission over time (B), and CDAI remission (C) over time. *Not all patients reached later timepoints at time of data analysis. CDAI remission is defined as $\leq 2.8$. SDAI remission is defined as $\leq 3.3$. As-observed analysis, based on patients entering the LTE who received at least 1 dose of abatacept. DAS28: 28 -joint Disease Activity Score; IV: intravenous; SC: subcutaneous; CDAI: Clinical Disease Activity Index; SDAI: Simplified Disease Activity Index; CRP: C-reactive protein; LTE: longterm extension.

\section{DISCUSSION}

In the DB period of the ACQUIRE trial, SC and IV ABA demonstrated comparable efficacy and safety over 6 months of treatment in patients with RA and inadequate response to MTX. The LTE of the ACQUIRE study demonstrates consistent safety and maintained clinical efficacy for patients who received open-label SC ABA up to a maximum of 44 months.

The overall safety profile of SC ABA during the LTE was consistent with the DB period; there were no new safety signals identified with increased exposure during the LTE. Results of our study support and extend findings from other phase II and III trials with SC ABA (125 mg/week) administered for up to 18 months $^{8,9,11}$. IR of SAE, serious infections, malignancies, and autoimmune events remained stable throughout the study when evaluated at 6-month intervals, and were not increased compared with the DB period. This is consistent with reports of safety events from 


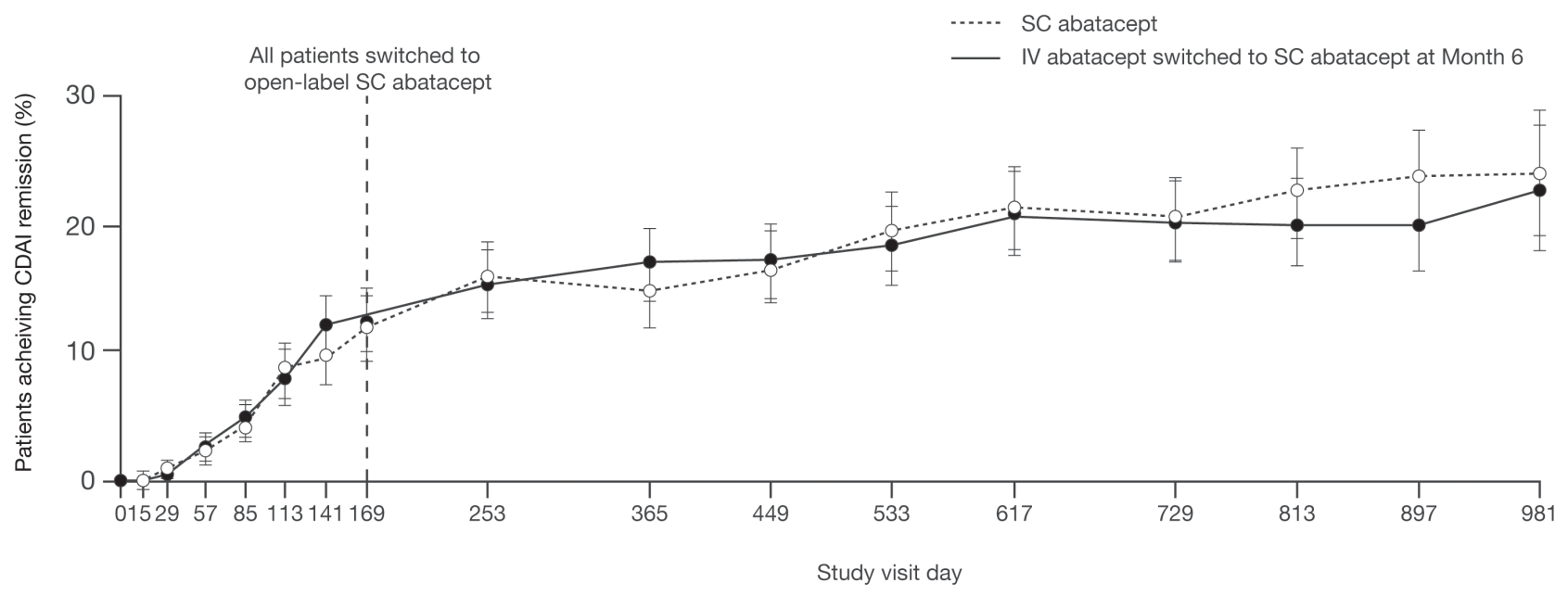

\begin{tabular}{|l|c|c|c|c|c|c|c|c|c|c|c|c|c|c|c|c|}
\hline & \multicolumn{10}{|c|}{ Patients with available data at each visit day* according to the original treatment group } \\
\cline { 2 - 28 } & 15 & 29 & 57 & 85 & 113 & 141 & 169 & 253 & 365 & 449 & 533 & 617 & 729 & 813 & 897 & 981 \\
\hline SC abatacept & 687 & 687 & 685 & 686 & 687 & 687 & 690 & 684 & 663 & 650 & 627 & 617 & 601 & 584 & 526 & 308 \\
\hline IV switched to SC abatacept & 660 & 668 & 670 & 671 & 670 & 669 & 671 & 665 & 654 & 633 & 620 & 607 & 585 & 575 & 515 & 301 \\
\hline
\end{tabular}

Figure 4. C.

the pooled SC ABA clinical trial experience of 1879 patients with 3086 patient-years of exposure, in which the IR of SAE evaluated at 6-month intervals did not increase with increasing ABA exposure $(9.25,95 \%$ CI $7.46,11.48 ; 10.75$, $95 \%$ CI $8.72,13.24 ; 8.21,95 \%$ CI $6.29,10.72 ; 8.85,95 \%$ CI $6.26,12.51$; and $12.06,95 \%$ CI $7.49,19.39)^{13,14}$. Moreover, the longterm safety profile seen here with $\mathrm{SC}$ ABA is similar to the consistent longterm profile demonstrated for IV ABA, which was reported for 4419 patients with more than 12,000 cumulative patient-years of exposure ${ }^{15}$, and demonstrated that IR of hospitalized infections $(3.35,2.63,2.31,2.33$, $1.55,2.48$, and 3.03 from years 1 to 7 , respectively) and malignancies $(0.62,0.55,0.81,0.81,0.99,0.53$, and 1.66 from years 1 to 7 , respectively) evaluated at 12-month intervals remained relatively stable with increasing $\mathrm{ABA}$ exposure.

The occurrence of malignancies and autoimmune events is of interest for longterm biologic treatment. The IR of overall malignancy reported in the ACQUIRE LTE study (1.19) is within the range of IR reported for cohorts of DMARD-treated patients with RA $(0.67-1.77)^{16}$. The IR reported here with SC ABA for breast and lung cancers ( 0.17 and 0.10 , respectively) were also within the range of IR reported for cohorts of DMARD-treated patients with RA $(0.14-0.34,0.09-0.26 \text {, respectively })^{16}$. The IR of different malignancies that occurred in the ACQUIRE LTE are also consistent with the IR reported for the pooled SC ABA clinical trial experience ( 0.46 for basal cell carcinoma, 0.16 each for breast cancer and squamous cell carcinoma of skin) ${ }^{14}$ and the pooled IV ABA clinical trial experience
$(0.15 \text { and } 0.12 \text { for lung and breast cancers, respectively })^{15}$. The IR of overall autoimmune events in the ACQUIRE study (1.31) and specific autoimmune events, including psoriasis $(0.31)$, were also consistent with IR reported for the pooled SC ABA clinical trial experience (1.28 and 0.29, respectively) ${ }^{14}$ and the pooled IV ABA clinical trial experience (1.99 and 0.57 , respectively $)^{15}$.

Four cases of TB $(0.14,95 \%$ CI $0.05,0.36)$ were reported, all of them in endemic areas, one of which was a patient with a history of TB. Two patients were PPD-negative at entry, and all were diagnosed with TB more than a year after initiation of ABA treatment. This is consistent with reports from the pooled IV ABA clinical trial experience, in which 8 cases of TB were reported, with an IR of $0.07 / 100$ patient-years ${ }^{15}$.

The frequency of injection-site reactions, including pain, was relatively low in the LTE and similar to that in the DB period, and events were generally mild. This is consistent with previous clinical trial reports of SC ABA use, and further demonstrates that the frequency of SC injection-site reactions is reduced with increasing treatment time ${ }^{8,9}$.

During the LTE, 21 deaths were recorded (IR 0.71, 95\% CI $0.44,1.09$ ), of which 7 were classified as being due to MI or sudden death. None of these events were classified as related to treatment by the investigators. This is consistent with the pooled IV ABA clinical trial experience, during which 73 deaths were reported at an IR of $0.60(95 \%$ CI $0.47,0.76$ ), including 26 due to cardiac disorders ${ }^{15,17}$. There are no available data to suggest an increased CV risk with ABA treatment of RA, with comparable proportions of

Personal non-commercial use only. The Journal of Rheumatology Copyright @ 2014 . All rights reserved. 
ABA- and placebo-treated patients experiencing serious cardiac disorders during the pooled IV ABA clinical trial experience (IR $1.42,95 \%$ CI $0.98,1.99$ and $2.24,95 \%$ CI $1.34,3.50)^{15}$.

Immunogenicity to SC ABA reported in this LTE was based on ECL analysis, and $\sim 10 \%$ of patients experienced an immunologic response either during treatment or following discontinuation. However, few patients (2.1\%) experienced persistent immunogenicity, and titers were generally low and transient, and did not increase with continued dosing. Further, immunogenicity did not appear to affect the safety or efficacy of SC ABA. Comparisons of data from this study with other ABA studies are problematic given immunogenicity has previously been evaluated using ELISA, rather than $\mathrm{ECL}^{7,8,9,10,11,18}$. There are a number of differences between the 2 assay types; notably, ECL has a greatly increased sensitivity compared to ELISA (12.2 $\mathrm{ng} / \mathrm{ml}$ vs $275 \mu \mathrm{g} / \mathrm{ml}$ ). However, the 6-month DB period of our study reported comparable rates of immunogenicity based on ELISA for SC and IV ABA-treated patients (1.1 vs $2.3 \%)^{12}$.

Safety and efficacy benefits were supported by high patient retention, with $\sim 83 \%$ of patients treated in the LTE remaining in the study at the time of assessment (up to a maximum of $3.5 \mathrm{yrs}$ ) and few discontinuations owing to lack of efficacy (4.9\%) over 33 months. This is comparable with longterm retention rates seen with IV ABA in similar patient populations; $82.3 \%$ and $90.9 \%$ of IV ABA-treated patients who continued treatment at Year 3 in the AIM study and at Year 2 of the ATTEST trial ${ }^{3,4}$. Retention rates observed in randomized clinical trials with anti-TNF (plus background DMARD) have ranged from $62 \%$ to $82 \%$ after 2 years, with $4 \%$ to $8 \%$ discontinuing as a result of lack of efficacy ${ }^{19,20,21,22}$.

The original DB period of ACQUIRE demonstrated comparable clinical and functional efficacy for SC and IV ABA over 6 months, with high proportions of patients achieving disease activity targets and clinically meaningful improvements in ACR and HAQ-DI scores. Longterm administration of SC ABA during the LTE, up to Day 981, was associated with maintained improvements in ACR and HAQ-DI responses for patients who continued taking treatment. In particular, the proportions of patients achieving disease activity targets of DAS28, SDAI, and CDAI remission were maintained through the LTE. Similar longterm outcomes have been reported for patients treated with IV ABA in a similar population of patients with RA and inadequate response to $\mathrm{MTX}^{3,5}$. Clinical and functional benefits were maintained longterm during the ACQUIRE study, regardless of whether patients received SC ABA throughout, or switched from IV ABA to SC ABA at the start of the LTE. These observations support findings from the ATTUNE study, which evaluated safety and efficacy in patients who switched from IV to SC $\mathrm{ABA}^{9}$. Together these observations suggest that patients can safely switch from a monthly $\sim 10 \mathrm{mg} / \mathrm{kg}$ dose of IV ABA to a fixed weekly 125 $\mathrm{mg}$ dose of SC ABA with maintained clinical efficacy.

Nearly all patients continued to receive concomitant MTX, and the mean dose remained stable throughout the LTE of the ACQUIRE study. Mean steroid dose remained consistent with mean dose at entry into the LTE. Further, the proportions of patients receiving steroids throughout this LTE is consistent with other ABA reports, including a head-to-head trial of SC ABA and adalimumab, in which 65.1 and $64.0 \%$ of patients received concomitant corticosteroids at any time over 2 years ${ }^{23}$. These proportions are consistent with reports of steroid use in clinical trials with other biologics, which have ranged from 67.9 to $50.4 \%$, although it is generally acknowledged that concomitant steroid dosing is not widely reported ${ }^{24}$.

There are limitations to our study and subsequent analysis that should be noted. In accordance with many other reports of LTE data, efficacy in our study is based on as-observed analyses, which include only patients with available data and can be vulnerable to discontinuations among those who do not respond as well to treatment. Further, the LTE was open-label in nature, which, although standard for longterm reporting, can result in bias of results toward improved efficacy outcomes (based on subjective reporting) and increased safety event reporting.

The findings from the LTE of the ACQUIRE study represent the largest and longest observation period for SC ABA treatment: up to 44 months for some patients. With continued longterm treatment, SC ABA was well tolerated with no increase in the incidence of safety events over time, consistent with previous findings ${ }^{13,14}$. SC ABA was associated with high patient retention and demonstrated maintained improvements in clinical and functional efficacy outcomes and disease activity targets.

\section{ACKNOWLEDGMENT}

The authors thank Eve Guichard, BSc (Hons), Caudex Medical, funded by Bristol-Myers Squibb, for her professional medical writing and editorial assistance.

\section{REFERENCES}

1. Bathon J, Robles M, Ximenes AC, Nayiager S, Wollenhaupt J, Durez $\mathrm{P}$, et al. Sustained disease remission and inhibition of radiographic progression in methotrexate-naive patients with rheumatoid arthritis and poor prognostic factors treated with abatacept: 2-year outcomes. Ann Rheum Dis 2011;70:1949-56.

2. Kremer JM, Genant HK, Moreland LW, Russell AS, Emery P, Abud-Mendoza C, et al. Results of a two-year followup study of patients with rheumatoid arthritis who received a combination of abatacept and methotrexate. Arthritis Rheum 2008;58:953-63.

3. Kremer JM, Russell AS, Emery P, Abud-Mendoza C, Szechinski J, Westhovens R, et al. Long-term safety, efficacy and inhibition of radiographic progression with abatacept treatment in patients with rheumatoid arthritis and an inadequate response to methotrexate: 3-year results from the AIM trial. Ann Rheum Dis 2011; 70:1826-30.

Personal non-commercial use only. The Journal of Rheumatology Copyright $(\subset) 2014$. All rights reserved. 
4. Schiff M, Keiserman M, Codding C, Songcharoen S, Berman A, Nayiager S, et al. Clinical response and tolerability to abatacept in patients with rheumatoid arthritis previously treated with infliximab or abatacept: open-label extension of the ATTEST Study. Ann Rheum Dis 2011;70:2003-7.

5. Westhovens R, Kremer JM, Moreland LW, Emery P, Russell AS, Li $T$, et al. Safety and efficacy of the selective costimulation modulator abatacept in patients with rheumatoid arthritis receiving background methotrexate: a 5-year extended phase IIB study. J Rheumatol 2009;36:736-42.

6. Genovese MC, Schiff M, Luggen M, Becker JC, Aranda R, Teng J, et al. Efficacy and safety of the selective co-stimulation modulator abatacept following 2 years of treatment in patients with rheumatoid arthritis and an inadequate response to anti-tumour necrosis factor therapy. Ann Rheum Dis 2008;67:547-54.

7. Corbo M, Valencia X, Raymond R, Summerill R, Agrawal S, Shergy $\mathrm{W}$, et al. A subcutaneous administration regimen for abatacept in patients with rheumatoid arthritis: pharmacokinetics, safety and immunogenicity [abstract]. Arthritis Rheum 2008;58 Suppl 9:S307.

8. Kaine J, Gladstein G, Strusberg I, Robles M, Louw I, Gujrathi S, et al. Evaluation of abatacept administered subcutaneously in adults with active rheumatoid arthritis: impact of withdrawal and reintroduction on immunogenicity, efficacy and safety (phase IIIb ALLOW study). Ann Rheum Dis 2012;71:38-44.

9. Keystone EC, Kremer JM, Russell A, Box J, Abud-Mendoza C, Elizondo MG, et al. Abatacept in subjects who switch from intravenous to subcutaneous therapy: results from the phase IIIb ATTUNE study. Ann Rheum Dis 2012;71:857-61.

10. Nash P, Nayiager S, Genovese M, Kivitz A, Oelke K, Ludivico C, et al. Immunogenicity is not increased with subcutaneous administration of abatacept with and without methotrexate in patients with rheumatoid arthritis: results from a phase III study. Arthritis Rheum 2009;60:S633-4

11. Nash P, Nayiager S, Genovese M, Rodriguez C, Delaet I, Elegbe A, et al. Low immunogenicity, consistent safety and sustained clinical efficacy over 18 months of subcutaneous administration of abatacept with and without methotrexate in patients with rheumatoid arthritis-results from a phase III study. Ann Rheum Dis 2010;69:iii97.

12. Genovese MC, Covarrubias A, Leon G, Mysler E, Keiserman M, Valente R, et al. Subcutaneous abatacept versus intravenous abatacept: a phase IIIb noninferiority study in patients with an inadequate response to methotrexate. Arthritis Rheum 2011;63:2854-64.

13. Alten R, Kaine J, Keystone EC, Nash P, Delaet I, Qi K, et al. Safety of subcutaneous abatacept in patients with rheumatoid arthritis (RA): Integrated analysis of five clinical trials up to 4.5 years. Ann Rheum Dis 2011;70 Suppl 3:617.
14. Alten R, Kaine J, Keystone EC, Nash P, Delaet I, Qi K, et al. Safety profile of subcutaneous abatacept focusing on clinically relevant events in patients with rheumatoid arthritis (RA) and up to 4.5 years of exposure. Arthritis Rheum 2011;63 Suppl 10:S150.

15. Weinblatt ME, Moreland LW, Westhovens R, Cohen RB, Kelly SM, Khan N, et al. Safety of abatacept administered intravenously in treatment of rheumatoid arthritis: integrated analyses of up to 8 years of treatment from the abatacept clinical trial program. J Rheumatol 2013;40:787-97.

16. Simon TA, Smitten AL, Franklin J, Askling J, Lacaille D, Wolfe F, et al. Malignancies in the rheumatoid arthritis abatacept clinical development programme: an epidemiological assessment. Ann Rheum Dis 2009;68:1819-26.

17. Weinblatt ME, Moreland L, Westhovens R, Cohen RB, Kelly SM, Khan N, et al. The safety of abatacept administered intravenously in the treatment of rheumatoid arthritis: integrated analyses of up to 8 years of treatment from the abatacept clinical trial program. J Rheumatol 2013;40:787-97.

18. Haggerty HG, Abbott MA, Reilly TP, DeVona DA, Gleason CR, Tay L, et al. Evaluation of immunogenicity of the T cell costimulation modulator abatacept in patients treated for rheumatoid arthritis. J Rheumatol 2007;34:2365-73.

19. Breedveld FC, Weisman MH, Kavanaugh AF, Cohen SB, Pavelka $\mathrm{K}$, van Vollenhoven $\mathrm{R}$, et al. The PREMIER study: A multicenter, randomized, double-blind clinical trial of combination therapy with adalimumab plus methotrexate versus methotrexate alone or adalimumab alone in patients with early, aggressive rheumatoid arthritis who had not had previous methotrexate treatment. Arthritis Rheum 2006;54:26-37.

20. Kremer JM, Weinblatt ME, Bankhurst AD, Bulpitt KJ, Fleischmann RM, Jackson CG, et al. Etanercept added to background methotrexate therapy in patients with rheumatoid arthritis: continued observations. Arthritis Rheum 2003;48:1493-9.

21. van der Heijde D, Klareskog L, Rodriguez-Valverde V, Codreanu C, Bolosiu H, Melo-Gomes J, et al. Comparison of etanercept and methotrexate, alone and combined, in the treatment of rheumatoid arthritis: two-year clinical and radiographic results from the TEMPO study, a double-blind, randomized trial. Arthritis Rheum 2006;54:1063-74.

22. Weinblatt ME, Keystone EC, Furst DE, Kavanaugh AF, Chartash EK, Segurado OG. Long term efficacy and safety of adalimumab plus methotrexate in patients with rheumatoid arthritis: ARMADA 4 year extended study. Ann Rheum Dis 2006;65:753-9.

23. Schiff M, Weinblatt ME, Valente R, van der Heijde D, Citera G, Elegbe A, et al. Head-to-head comparison of subcutaneous abatacept versus adalimumab for rheumatoid arthritis: two-year efficacy and safety findings from AMPLE trial. Ann Rheum Dis 2014;73:86-94.

24. Alten RH. Clinical trials: Insufficient data on glucocorticoid use in RA trials. Nat Rev Rheumatol 2011;7:318-9. 